\title{
DECELERATION BY DYNAMICAL FRICTION IN A GASEOUS MEDIUM
}

\author{
F. J. Sánchez-Salcedo and A. Brandenburg \\ Department of Mathematics, University of Newcastle, Newcastle upon Tyne, NE1 7RU, UK; \\ f.j.sanchez-salcedo@ncl.ac.uk, axel.brandenburg@ncl.ac.uk \\ Received 1999 May 4; accepted 1999 July 6; published 1999 August 3
}

\begin{abstract}
The drag force experienced by a gravitational body moving in a straight-line trajectory through a homogeneous isothermal gaseous medium of given sound speed is investigated numerically. For perturbers with constant velocity, linear theory describes successfully the temporal evolution and magnitude of the force. The result obtained recently by E. Ostriker-that for Mach numbers $\mathcal{M}=1-2$ the force is stronger in a gaseous medium than in a collisionless medium, as described by the standard Chandrasekhar formula-is confirmed. The corresponding minimum impact radius $r_{\min }$ for a body described with a Plummer model with core radius $R_{\text {soft }}$ is $r_{\text {min }} / R_{\text {soft }} \approx$ 2.25. When $\mathcal{M}<1$, the drag force is strongly suppressed, which is consistent with Ostriker's results but in disagreement with the Chandrasekhar formula. However, when the perturber is decelerated by its own wake to $\mathcal{M}<1$, the effective drag force remains initially somewhat larger than the value in the case of constant velocity because it takes some time to get rid of the wake that was generated during its supersonic history.
\end{abstract}

Subject headings: galaxies: kinematics and dynamics — galaxies: star clusters — hydrodynamics — waves

\section{INTRODUCTION}

The Chandrasekhar dynamical friction formula, although simple, provides an accurate estimate of the drag experienced by a perturber moving in a stellar system. The variety of approximations introduced by Chandrasekhar (1943) has been widely discussed in the literature (e.g., Binney \& Tremaine 1987).

The hydrodynamical friction, i.e., the gravitational interaction of a massive object with its own wake in a (homogeneous) gaseous medium, can be calculated more easily because of the closure of the fluid equations with a given equation of state. It turns out that accurate determinations of the dynamical friction force in a gaseous medium may shed light also on the problem of the time evolution of the Coulomb logarithm in a collisionless background. Also, the fact that for Mach numbers $\mathcal{M} \ll 1$ the gaseous friction force is nonvanishing is an indication that the collisionless dynamical friction may be nonzero even in the absence of background particles slower than the perturber. Previous theoretical work appears to be inconclusive (e.g., Zamir 1992).

Recently, Ostriker (1999) has computed the hydrodynamical friction, for both subsonic and supersonic cases, as the gravitational force between the perturber with constant velocity and the perturbed density which was calculated in linear perturbation theory. In this approach, the force upon a particle of mass $M_{p}$ traveling at velocity $-V \hat{z}(V>0)$ in a medium of undisturbed density $\rho_{0}$ and sound speed $c_{s}$ is

$$
F_{\mathrm{df}}=\frac{4 \pi G^{2} M_{p}^{2} \rho_{0}}{V^{2}}\left[\frac{1}{2} \ln \left(\frac{1+\mathcal{M}}{1-\mathcal{M}}\right)-\mathcal{M}\right]
$$

for $\mathcal{M} \equiv V / c_{s}<1$ and $t>r_{\min } /\left(c_{s}-V\right)$ and

$$
F_{\mathrm{df}}=\frac{4 \pi G^{2} M_{p}^{2} \rho_{0}}{V^{2}}\left[\frac{1}{2} \ln \left(1-\mathcal{M}^{-2}\right)+\ln \left(\frac{V t}{r_{\text {min }}}\right)\right]
$$

for $\mathcal{M}>1$ and $t>r_{\min } /\left(V-c_{s}\right)$. The perturber is assumed to be formed at $t=0$. The minimum radius $r_{\min }$ is adopted in order to regularize the divergence of the gravitational potential of a point mass.

Interestingly, Ostriker concludes that the drag is more efficient in a gaseous medium by a factor 2-4 than the friction given by the classical Chandrasekhar formula provided $\mathcal{M}=$ 1-2 and also that the drag is nonvanishing when $\mathcal{M}<1$. The relatively stronger friction in the gaseous supersonic case may have relevant consequences in different scenarios such as the rate of orbital decay of globular clusters and primordial black holes toward the centers of galaxies, the relaxation of stellar protoclusters (see also Gorti \& Bhatt 1996 and Saiyadpour, Deiss, \& Kegel 1997), or the migration of protoplanets. She points out that perturbers moving at speeds near $\mathcal{M}=1$ resonantly interact with the pressure waves that they launch in the medium. The inclusion of any possible resonance is crucial for an accurate determination of the dynamical friction force. One could argue that this enhancement of the computed force might be an artifact of the linearized fluid equations being unable to capture the surroundings of the Mach cone which have $\rho<\rho_{0}$. These surroundings might give a nonnegligible contribution to the gravitational drag force when integrating over the whole volume. Our aim is to solve the nonlinear equations in order to capture properly both effects. We shall see that the second effect is unimportant. In addition, we include the back-reaction of the background onto the motion of the object. In fact, in many cases of interest, a perturber may suffer an appreciable deceleration and therefore it can no longer be assumed to be moving at constant velocity; instead, it may rapidly shift, for instance, from the supersonic regime to the subsonic one for which the hydrodynamical friction force is strongly suppressed.

\section{ESTIMATING THE GRAVITATIONAL DRAG: NUMERICAL RESULTS}

We present here numerical simulations of the disturbances produced in the gas by a gravitational perturber. The first aim is to obtain the force of dynamical friction as a function of $\mathcal{M}$ and $t$. Although the far-field contribution to friction is limited by the finiteness of the domain of the simulation, we are able 
to compare the numerical gravitational wake with that predicted by the linearized fluid equations.

For the sake of simplicity, we adopt an isothermal gas, i.e., $P=c_{s}^{2} \rho$. Using an explicit code that is sixth order in space and third order in time, we solve numerically the continuity and Euler equations for an isothermal compressible gas:

$$
\begin{aligned}
& \frac{\partial \rho}{\partial t}+\nabla \cdot(\rho \boldsymbol{v})=0, \\
& \frac{\partial \boldsymbol{v}}{\partial t}+(\boldsymbol{v} \cdot \boldsymbol{\nabla}) \boldsymbol{v}=-\frac{1}{\rho} \boldsymbol{\nabla} P-\nabla \phi_{p},
\end{aligned}
$$

where $\phi_{p}(|\boldsymbol{r}-\boldsymbol{X}(t)|)$ is the gravitational potential generated by the perturber at the position $\boldsymbol{X}(t)$. For numerical purposes, an artificial viscosity term $\nu \nabla^{2} \boldsymbol{v}$ has been introduced in the momentum equations. Because of the axial symmetry of the problem, we may use cylindrical coordinates $(R, z)$ with $R$ the usual cylindrical radius.

We consider a finite domain of size $R \in\left[0, L_{R, \max }\right]$ and $z \in\left[L_{z, \min }, L_{z, \max }\right]$ with open boundary conditions for $z=$ $L_{z, \min }, z=L_{z, \max }$, and $R=L_{R}$. The size of the domain was taken large enough to ensure that the perturbed density does not leave the box (see Table 1). In addition, a nonuniform mesh was implemented to optimize resolution near the origin.

The gravitational potential produced by the perturber was smoothed at short distances with a softening radius $R_{\text {soft }}$, following a Plummer model, $\phi_{p}=-G M_{p} /\left(r^{2}+R_{\text {soft }}^{2}\right)^{1 / 2}$. The introduction of a smoothed potential contributes to regularizing the singularity of the density in the Mach cone. For this reason one expects a slight dependence of $r_{\text {min }}$ on $\mathcal{M}$. The relation between $r_{\min }$ and $R_{\text {soft }}$ will be determined empirically. In all the experiments considered below, $R_{\text {soft }}$ was taken to be at least 20 times larger than the accretion radius $R_{\mathrm{ac}} \equiv 2 G M_{p} / V^{2}$, a condition that prevents the generation of a gravitational shock. Runs with $R_{\text {ac }}>R_{\text {soft }}$ were carried out, but the value of the dynamical friction was not significantly effected by that.

The drag force on the body was calculated from the density distribution

$$
\boldsymbol{F}_{\mathrm{df}}=\int \rho \boldsymbol{\nabla} \boldsymbol{\phi}_{p} d^{3} \boldsymbol{r}
$$

and also from the total momentum transmitted to the fluid per unit time. The agreement between them provides a test of the consistency of the results. In the next subsection the dynamical friction force $F_{\mathrm{df}}(t)$ is computed assuming a constant velocity of the perturber, i.e., $\boldsymbol{X}(t)=\boldsymbol{X}_{0}+\boldsymbol{V} t$. Any resonant effect or nonlinear signature should be fully captured in our simulations.

\subsection{Drag Force for Constant-Velocity Perturbers}

Gas density profiles and velocity fields are presented in Figure 1 for three cases: subsonic $(\mathcal{M}=0.3)$, transonic $(\mathcal{M}=$ $1.0)$, and supersonic $(\mathcal{M}=1.4)$ at $t=1.5$. The length and velocity units are such that $\mathcal{M}^{2} R_{\mathrm{ac}}=1$ and $c_{s}=1000$. In these units, the adopted value for the softening radius was $R_{\text {soft }}=$ 20 . The number of grid points in the radial and vertical direction, $N_{R}$ and $N_{z}$ respectively, are given in Table 1. We see from Figure 2 that the dynamical friction force saturates very quickly for subsonic perturbers, but increases with time for supersonic ones, in good agreement with the linear hydrodynamic theory. From Figure 3, it is apparent that good fits for the supersonic
TABLE 1

RESOLUTION

\begin{tabular}{cccccccc}
\hline \hline $\mathcal{M}$ & $L_{z, \min }$ & $L_{z, \max }$ & $L_{R, \max }$ & $N_{R}$ & $N_{z}$ & $\begin{array}{c}\nu /\left(\delta x c_{s}\right) \\
\left(\times 10^{-2}\right)\end{array}$ & $\begin{array}{c}F_{\mathrm{df}} / F_{0} \\
\text { at } t=1.5\end{array}$ \\
\hline $0.3 \ldots \ldots \ldots$ & -3700 & 3700 & 3700 & 200 & 400 & 4.2 & 0.106 \\
$0.75 \ldots \ldots$. & -3700 & 3700 & 3700 & 200 & 400 & 4.2 & 0.398 \\
$0.9 \ldots \ldots \ldots$ & -3700 & 3700 & 3700 & 200 & 400 & 4.2 & 0.709 \\
$1.0 \ldots \ldots \ldots$ & -3700 & 3700 & 3700 & 500 & 700 & 21.0 & 1.542 \\
$1.05 \ldots \ldots \ldots$ & -3700 & 3700 & 3700 & 500 & 700 & 21.0 & 2.110 \\
$1.12 \ldots \ldots \ldots$ & -1000 & 3700 & 3700 & 200 & 400 & 4.9 & 2.277 \\
& -3700 & 3700 & 3700 & 200 & 400 & 4.2 & 2.265 \\
& -3700 & 3700 & 3700 & 200 & 400 & 1.0 & 2.272 \\
$1.12^{\mathrm{a}} \ldots \ldots$ & -1000 & 3700 & 3700 & 300 & 600 & 7.4 & 2.278 \\
$1.3 \ldots \ldots \ldots$ & -2300 & 3700 & 3700 & 200 & 400 & 4.2 & 2.280 \\
$2.0 \ldots \ldots \ldots$ & -1150 & 7500 & 3700 & 200 & 400 & 4.2 & 1.997 \\
& -1150 & 7500 & 3700 & 400 & 600 & 4.2 & 0.969 \\
$2.0^{\mathrm{a}} \ldots \ldots \ldots$ & -1150 & 7500 & 3700 & 200 & 600 & 8.4 & 0.970 \\
\end{tabular}

Note. - The body is localized at $(R=0, z=0) ; \delta x$ is the mesh width at the origin, and $F_{0}=4 \pi G^{2} M_{p}^{2} \rho_{0} / c_{s}^{2}$.

${ }^{a}$ Numerical experiment in linear theory.

runs correspond to identifying the minimum radius adopted in the linear theory as $r_{\text {min }} \simeq 2.25 R_{\text {soft }}$. This value may be compared with the inferred one in stellar systems (White 1976). For the potential described by a Plummer model, the minimum stellar radius according to White (1976) is $r_{\text {min }}^{*} \simeq \sqrt{e} R_{\text {soft }} \simeq 1.65 R_{\text {soft }}$, which differs somewhat from the value of $r_{\min }$ for a gaseous medium obtained here numerically. For the subsonic cases, for which the linear theory predicts a time-independent force, the time evolution is fitted well by a function of the form $F_{\infty}\left[1-\exp \left(-t / t_{\text {sub }}\right)\right]$, where $t_{\text {sub }} \approx 0.06$.

In Figure 4 the value of the dynamical drag force obtained in our simulations at $t=t_{\max }$ is plotted as symbols versus Mach number, where $t_{\max }$ is the time of the run. The strongest average force occurs when the body is moving at $\mathcal{M} \simeq 1.12$. The continuous line, which corresponds to the curve predicted by linear analysis with $r_{\min }=2.25 R_{\text {soft }}$, matches the data reasonably well. We find only a weak dependence of the results on the numerical resolution $(<0.6 \%)$, extent of the computational domain $(<0.5 \%)$, and the value of the numerical viscosity $(<0.3 \%)$. The largest dependence $(\simeq 1 \%)$ results if we artificially omit the nonlinear terms in the numerical solution. The good agreement between linear theory and numerical results is a confirmation that the gas dynamics is well described by the linear approximation in the regions in which most of the contribution to dynamical friction come from. The small deviations suggest a slight dependence of $r_{\min }$ on the Mach number, as discussed above.

\subsection{Drag Force for Decelerating Perturbers}

Dynamical friction becomes of great interest for times long enough such that the perturber changes appreciably its orbital energy. Here we only consider a perturber moving freely in a straight-line orbit. Let us ask the question, How long does a perturber take to decrease its velocity to half its initial velocity? For perturbers with initial $\mathcal{M}=1-2$, it is far from clear whether the average force is more effective in a gaseous medium than that obtained by applying the plain Chandrasekhar formula. In Figure 5 we plot the velocity of the perturber with time for both situations. The unperturbed gas density has been chosen large enough to have significant drag in the period of the run of the simulation. Although $\rho_{0}$ is then extremely large to be representative of any astrophysical scenario and also the assumption of negligible self-gravity would be no longer valid, 

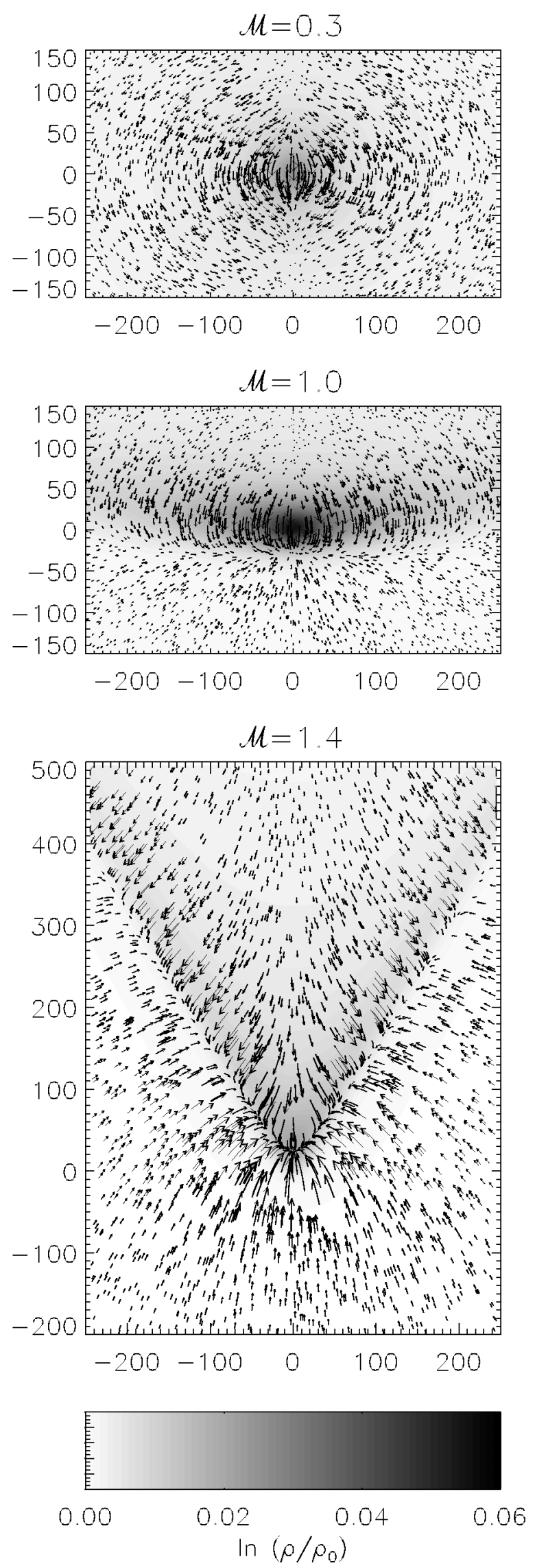

FIG. 1.-Gray-scale plot of $\ln \left(\rho / \rho_{0}\right)$ together with velocity vectors for three different values of $\mathcal{M}$.

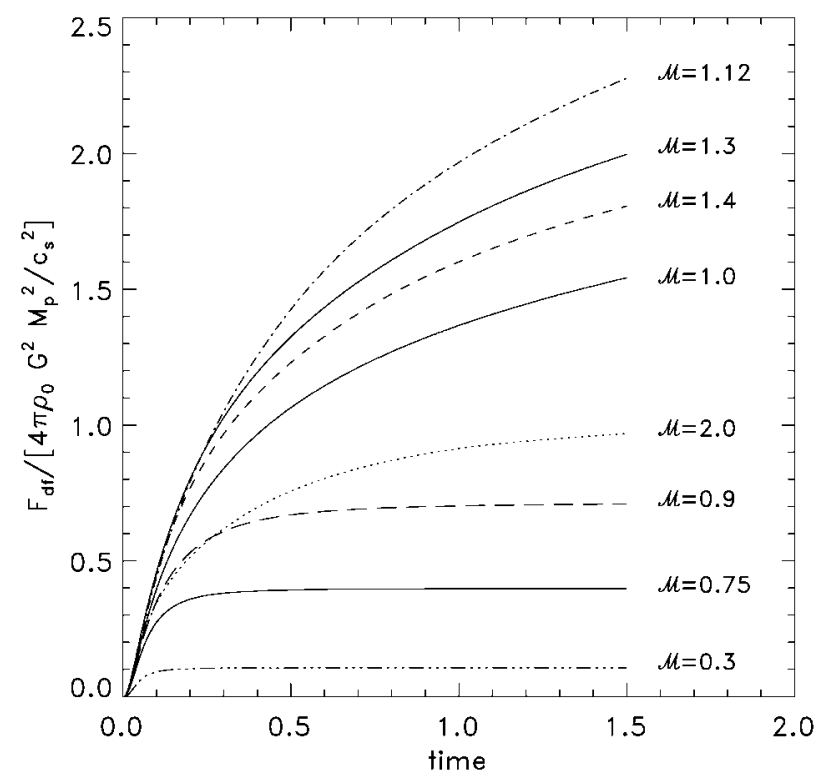

FIG. 2.-Time evolution of the force for constant-velocity perturbers

it illustrates properly the behavior of the drag experienced by the perturber. In order to compare with the values given by Chandrasekhar's formula, the Coulomb logarithm was assumed to be time-independent, as widely used in many different scenarios, with a value $\ln \Lambda=\ln \left(r_{\max } / r_{\min }\right) \sim \ln \left(c_{\mathrm{s}} t_{\max } / 2 R_{\text {soft }}\right)$. For initial Mach numbers less than $\sim 1.2$ the friction force drops strongly after $\mathcal{M}=1$, and consequently the Chandrasekhar formula overestimates the friction. If the Mach number is larger than 1.4, the Chandrasekhar formula underestimates the gravitational drag, but not dramatically (see Fig. 5). These arguments cannot be extended to perturbers subject to an external force. From Figure 5 we see that, for $\mathcal{M}>1$, the velocity of the body is well described by integrating the force according

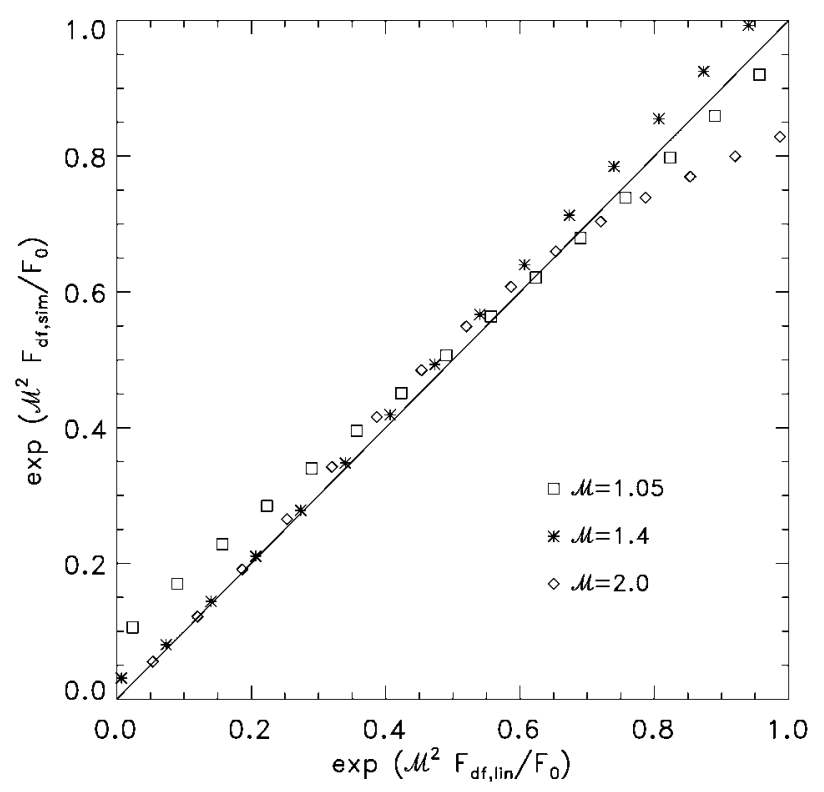

FIG. 3.-Comparison of the force obtained by numerical simulation with that predicted by linear theory with $r_{\min }=45$ for different values of $\mathcal{M}>1$. The values on the abscissa have been normalized to the maximum. The diagonal is shown as a solid line and $F_{0}=4 \pi G^{2} M_{p}^{2} \rho_{0} / c_{s}^{2}$. 


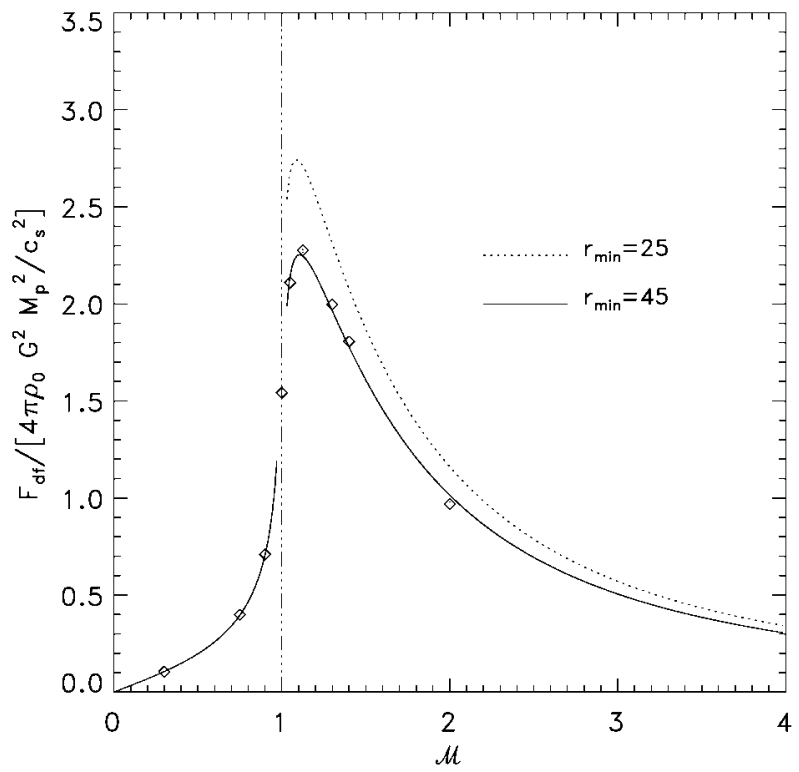

FIG. 4.-Gravitational drag force at $t=1.5 \mathrm{vs}$. Mach number. The numerical results are plotted with symbols. The curves correspond to the predictions by the linear theory (Ostriker 1999) for different values of $r_{\min }$.

to the equation

$$
\frac{d \mathcal{M}}{d t}=-\frac{F_{\mathrm{df}}(\mathcal{M}, t)}{c_{s}} .
$$

However, for $\mathcal{M}<1$ the actual deceleration is much larger than that obtained from equation (6). This is because of the memory of the wake behind the body. Its omission becomes most important just after passing the maximum in $F_{\text {df }}$ near $\mathcal{M} \approx 1.1$ (see Fig. 4).

\section{CONCLUSIONS}

As stressed by Ostriker (1999), the relatively stronger friction in the gaseous case may have relevant consequences in different

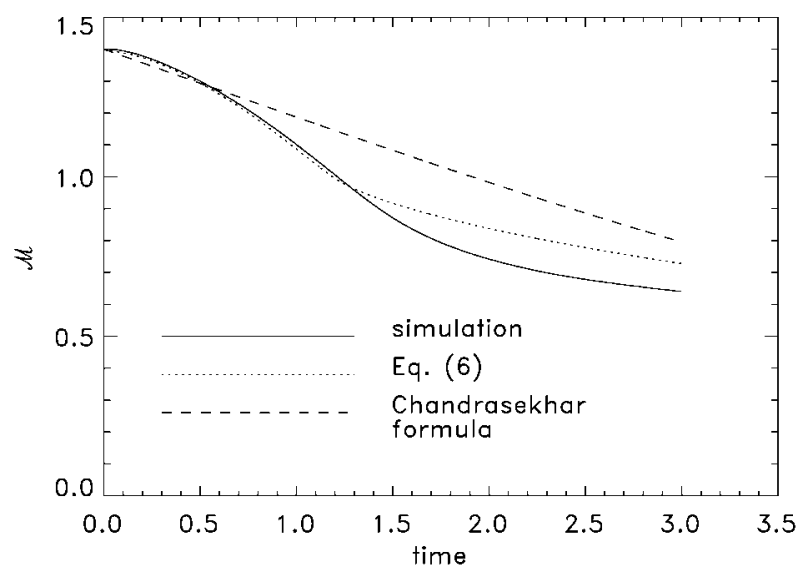

FIG. 5.-Deceleration of the body in a medium with no external forces (continuous line). For comparison, the time dependence of the perturber velocity by using the Chandrasekhar formula is also shown (dashed line) and by using the force in linear theory and eq. (6) (dotted line).

scenarios (see also $\S 1$ ). Here we have solved numerically the hydrodynamical equations including self-consistently the deceleration of the body. The main conclusion is that the linear approximation is reasonably accurate and may be applied successfully in future works for estimating the dynamical friction force. The expression for the force is also valid even if the radius of the perturber is smaller than the accretion radius. However, when passing through $\mathcal{M} \approx 1.1$, a decelerating body experiences an enhanced drag force due to a memory effect. This is because it takes some time for the body to get rid of the strong gravitational wake that has grown during the supersonic history.

We thank E. Ostriker for refereeing this Letter and making useful suggestions. We have also benefited from discussions with W. Dobler, B. v. Rekowski, and A. Shukurov. F. J. S. S. was supported by a TMR Marie Curie grant from the European Commission.

\section{REFERENCES}

Binney, J., \& Tremaine, S. 1987, Galactic Dynamics (Princeton: Princeton Univ. Press)

Chandrasekhar, S. 1943, ApJ, 97, 255

Gorti, U., \& Bhatt, H. C. 1996, MNRAS, 278, 611
Ostriker, E. C. 1999, ApJ, 513, 252

Saiyadpour, A., Deiss, B. M., \& Kegel, W. H. 1997, A\&A, 322, 756

White, S. D. M. 1976, MNRAS, 174, 467

Zamir, R. 1992, ApJ, 392, 65 